Bio-grafía: Escritos sobre la Biologia y su Enseñanza Vol. 4 No7. ISSN 2027-1034.

Segundo Semestre de 2011. P. p.121-131

\title{
EXPERIENCIAS SIGNIFICATIVAS COMO UN ACERCAMIENTO AL ESTUDIO DE QUIRÓPTEROS EN EL MUNICIPIO DE TOCANCIPA
}

SIGNIFICANT EXPERIENCES AS AN APPROACH TO STUDY MUNICIPALITY OF CHIROPTERA TOCANCIPA

Recibido: 17-08-2011

Aceptado: 14-12-2011

\section{Por: Clara Posso', Camilo Martín ${ }^{2}$}

El surgimiento de grupos de investigación en el aula de clase, son el resultado de las preguntas, observaciones y experiencias de los estudiantes que aparecen desde la exposición de las temáticas impartidas en clase por parte del docente que en la mayoría de los casos no son tenidas en cuenta, debido al poco interés del mismo en desarrollarlas para así poder fomentar los trabajos de investigación. Además se ha convertido en un acto rutinario hacer caso omiso de aquellas preguntas que requieren ser profundizadas para llegar a dar una posible solución, esto se debe a que los docentes han desarrollado una tendencia a generalizar de forma errónea las dudas, inquietudes y cuestionamientos de sus estudiantes, pasando inadvertido el interés y la duda que hace parte de su desarrollo intelectual, por otra parte como un agravante más los docentes en la mayoría de los casos tan solo se limitan a dar una breve explicación basada en supuestos que terminan por convertirse en conjeturas sin resolver, olvidadas inmediatamente concluye la clase de Biología.

En consecuencia, se formuló una propuesta para la búsqueda de nuevas metodologías para la comprensión de las temáticas acerca de la enseñanza de la biología y la práctica del saber investigativo, la docente del área de ciencias naturales decidió dar inicio a un pequeño semillero de exploración Biológica como parte de una alternativa para resolver las preguntas que surgieron a lo largo de las clases.

\footnotetext{
${ }^{1}$ Estudiante del postgrado en Enseñanza de la biología en Colombia. Universidad Pedagógica Nacional. clerposs3@yahoo.es

2 Estudiante de sexto semestre de Licenciatura en Biología. Universidad Pedagógica Nacional. Camilo co martin@hotmail.com
} 


\section{LOS INICIOS DE INDUCIENCIAS.}

El trabajo fue efectuado durante el año 2010 en la I.E.D Técnico Industrial, del municipio de Tocancipá, ubicado en el departamento de Cundinamarca a 1 hora de Bogotá D.C. Lugar donde se desarrolla este proyecto, que surgió como respuesta a las preguntas de investigación formuladas por el grupo INDUCIENCIAS conformado por 14 estudiantes, los cuales tenían edades entre 12 a 16 años, y a su vez pertenecían a los grados sexto, séptimo, octavo y decimo de Educación Básica secundaria, y habitan tanto el área rural como del casco urbano municipal y con una estratificación social de 2 y 3 (observar imagen 1,a).

El grupo se originó gracias a las inquietudes e indagaciones formuladas en varias clases de biología de grado octavo, las cuales llamaron la atención no solo de los estudiantes sino de su docente, quien gracias a su motivación y esfuerzo conllevaron a la necesidad de tomar tiempo extra clase para así poder dar una posible explicación a las dudas que inquietaban la mente de los niños acerca de la fauna y flora de su entorno. INDUCIENCIAS fue el nombre acuñado por el grupo debido a que sus actividades contribuyen a Inducir constantemente la curiosidad científica (observar imagen 1 , b).

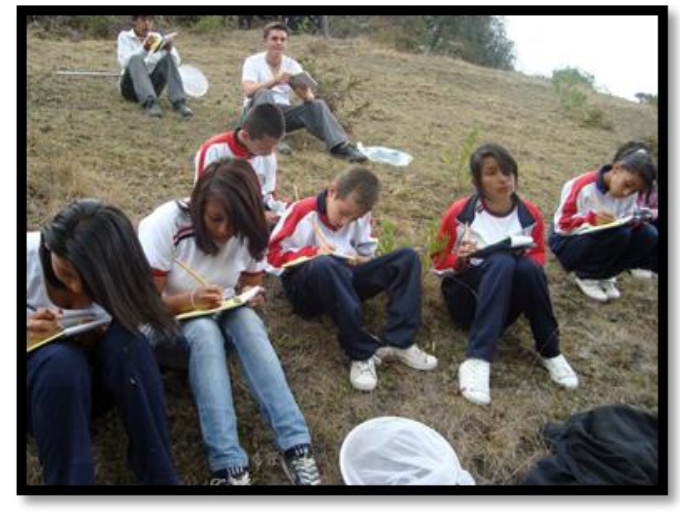

(1a)

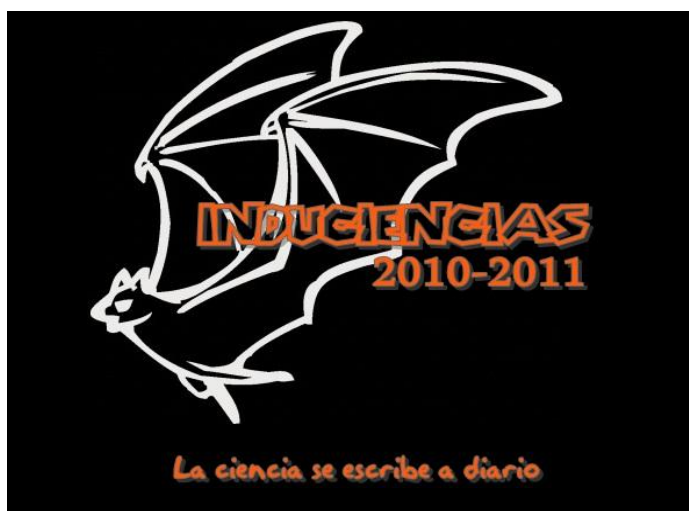

(1b)

Imagen 1, a: jóvenes que hicieron parte del Grupo investigador INDUCIENCIAS, con estratificación social 1, 2,3, además de pertenecer a distintos grados de básica secundaría.

Imagen 1, b: El nombre de INDUCIANCIAS, propuesto por los miembros del grupo investigador, junto a una imagen característica y su lema "la ciencia se escribe a diario".

\section{LAS CONJETURAS.}

Como punto de partida se tuvieron en cuenta las preguntas más frecuentes elaboradas por los estudiantes de grado octavo, sobre los sistemas vivientes que habitan los alrededores del colegio, esto con el objetivo de propiciar un acercamiento a la fauna y flora local, para así comprender las relaciones existentes entre los organismos y el impacto ambiental ocasionado al ecosistema por el depósito de residuos sólidos y las diferentes actividades humanas, esto conllevó al fortalecimiento del semillero de 
exploración Biológica ya existente en la Institución educativa, conformando un grupo que pudo dar sus primeros pasos a la ciencia, con un proyecto de investigación en el que las preguntas de los estudiantes surgidas a partir de sus observaciones fueron tenidas en cuenta para la investigación, pues ellos las consideraron fascinantes y la docente discurrió en que fueron de gran interés para conocer y describir especies posiblemente "nuevas" para el conocimiento de los niños, dándose lugar a una profundización en las temáticas que permitieron la aproximación al reconocimiento inicial de las clases y ordenes de la fauna y flora de Tocancipá.

Es así que de un número aproximado de mil cuestionamientos planteados acerca de la biodiversidad de la región, se eligieron aquellos que posiblemente producirían un impacto en la población, y que a través de su corroboración posibilitarían dar respuesta a las principales inquietudes manifestadas por los estudiantes, además de aportar información más precisa a las personas, para que tuviesen un mayor conocimiento de los organismos con los que comparten el municipio de Tocancipá. Algunas de estas preguntas fueron: ¿qué organismos comparten nuestro ambiente?, ¿Cuáles desconocemos? y ¿cómo las actividades humanas han puesto en peligro la permanencia de estos en su hábitat?

Como una fase inicial se tomaron en cuenta preguntas tales como; ¿Existirá en nuestra región alguna especie desconocida ya sea animal o vegetal?, ¿Están descritas todas las especies que habitan esta región de la sabana norte? Pero la pregunta que actuó como eje central para la presente investigación fue: ¿Hay murciélagos en Tocancipá?, Es pertinente resaltar que junto a esta pregunta se tuvieron en cuenta nuevos cuestionamientos a partir de los cuales fue posible desarrollar una investigación más profunda, tales preguntas fueron; ¿A qué familias pertenecen los murciélagos de Tocancipá?, ¿Cuál es su base alimenticia?, ¿Cuáles son sus hábitos nocturnos?, ¿Cuál es la flora y fauna asociada a estos individuos y por qué se relacionan con estos?, ¿Por qué se han encontrado murciélagos muertos en el colegio?, ¿Qué es lo que hace que vengan a sobrevolar la zona del colegio?, ¿Se está destruyendo su hábitat?, ¿Cuánto miden?, ¿Cómo se capturan?, ¿En dónde están sus refugios o cuevas?, ¿Cuáles son las especies más representativas de murciélagos en Tocancipá?, ¿Cuál es su índice poblacional?

Es necesario poner de manifiesto que ninguno de los integrantes del grupo INDUCIENCIAS tenía algún conocimiento estructurado acerca del orden Chiroptera y más aún ninguno sabía cómo abordar un trabajo de investigación biológica con estos organismos, por esta razón fue necesario basar el trabajo de investigación en la búsqueda de antecedentes que hicieran posible dirigir las actividades que permitieran construir conocimiento de manera rigurosa para dar un aporte de tipo científico a la comunidad de Tocancipá y así las personas puedan reconocer a los murciélagos, con el fin de modificar las concepciones erróneas que tuviesen acerca de ellos.

\section{VISITA A LAS CUEVAS DE TOCANCIPA.}

Como una segunda fase para abarcar el trabajo investigativo se diseñó un cronograma de actividades con metodologías pedagógicas así como científicas que facilitaron la resolución de la problemática establecida. Dentro de las actividades más importantes se destacaron las Salidas de Campo donde los estudiantes hicieron un reconocimiento de los sitios donde era posible encontrar murciélagos, cabe anotar que había desconocimiento sobre sus hábitats más frecuentados, tanto así que dos de los tres 
lugares visitados en donde se hallaron murciélagos fueron nombrados por los integrantes del grupo INDUCIENCIAS, porque carecían de nombre en la cartografía municipal.

Se obtuvieron resultados, como la plena ubicación de los diferentes hábitats donde se desarrollan y viven los murciélagos en Tocancipá - Cundinamarca, ya que se encontraron poblaciones de quirópteros en 2 de 3 cuevas de una red aun mayor de cuevas interconectadas con pasajes de difícil acceso para el grupo investigador en las veredas de La Fuente y Canavita, caracterizadas por estar en zonas de una altitud aproximada de $2800 \mathrm{msnm}$, con temperaturas promedio de $16^{\circ} \mathrm{C}$ en días soleados y $13^{\circ} \mathrm{C}$ en temporada invernal. La vegetación es propia de un bosque húmedo Andino donde se encuentran plantas como Musgos (clase: Briopsida), Hepáticas (clase: Hepaticopsida), Helechos (Orden: Órnales) Bromeliáceas (familia: Bromeliaceae), y Robles (Orden: Fagales) entre otras que aún están por determinar. Por otra parte la fauna asociada está caracterizada por la presencia de mamíferos pequeños como Zarigüeyas, (Orden: Didelphimorphia), ratas (Orden: Rodentia), aves copetonas (Orden: Passeriformes) e insectos tales como escarabajos (orden: Coleóptera).

Basados en lo anterior, el grupo investigador continuó con el análisis de las estructuras físicas de las cuevas y las características propias que posibilitan el hallazgo de murciélagos en estos lugares, en primer lugar se logró establecer que los procesos de formación de las cuevas fueron generados por la erosión de los suelos debido a las corrientes fluviales que formaban parte del relieve, con el transcurso del tiempo y tras sequias dejaron como consecuencia grandes grietas y profundos abismos que resultaron atractivos para los Quirópteros, de esta forma se asentaron allí y no solo por la estructura sino la facilidad y cercanía a su posible sustento alimenticio.

Se hicieron seis salidas de campo, en las diferentes veredas municipales, las primeras salidas se efectuaron como reconocimiento de la zona indagando a sus pobladores si tenían conocimiento de la presencia de murciélagos, si los han visto sobrevolar sus casas o si sabían de su existencia en dicho lugar, las posteriores salidas se consideraron en dicho proceso como un esfuerzo de equipo y el éxito logrado en los primeros hallazgos como una alegría propia y la motivación indispensable para continuar.

El primer lugar donde fueron hallados los murciélagos fue La Vereda Canavita, en la "Cueva del Lago" (observar imagen 2), acuñada así por el grupo, gracias a la presencia de un bello lago subterráneo que la caracteriza, además ésta presentaba una constante filtración de agua proveniente de la lluvia muy común en esta zona de bosque húmedo andino, ya como una breve descripción física de la cueva, se encontró en este un lugar con una humedad relativa del $100 \%$, sus paredes estaban cubiertas con sales minerales producto de la filtración por el constante goteo del agua proveniente del exterior. Había presencia de canales que según el grupo investigador propuso, eran las rutas de navegación de los murciélagos a sus distintas cámaras de reposo o al medio exterior para la búsqueda de alimento, por otra parte, la diversidad de organismos asociados se caracterizaba por la presencia de Artrópodos tales como Arácnidos e Insectos (observar imagen 3a y 3b), según lo observado presentaban adaptaciones para la vida en la oscuridad, ya que sus órganos sensoriales no eran ojos u ocelos sino por el contrario antenas y vellos recubriendo el cuerpo dando cuenta de lo dicho 
anteriormente. También se halló un hongo determinado como Histoplasma Sp.n. capsulatum (parecido a Capsulatum), de acuerdo a las investigaciones posteriores se estableció como un organismo asociado a lugares de alta concentración de humedad y oscuridad muy propias de las cuevas debido a su característico olor, para lo cual la recomendación impartida a los integrantes del grupo en posteriores visitas fue el uso constante del tapabocas.

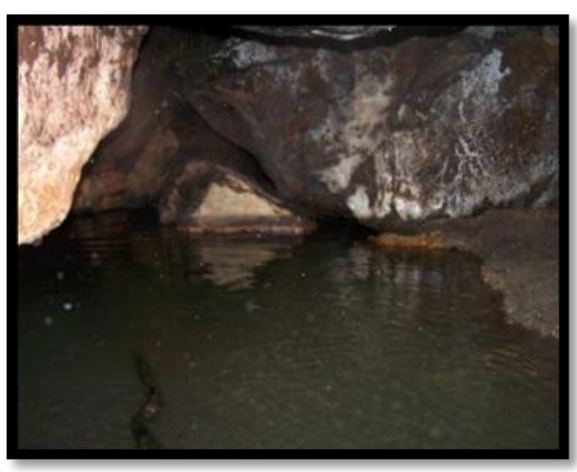

(2a)

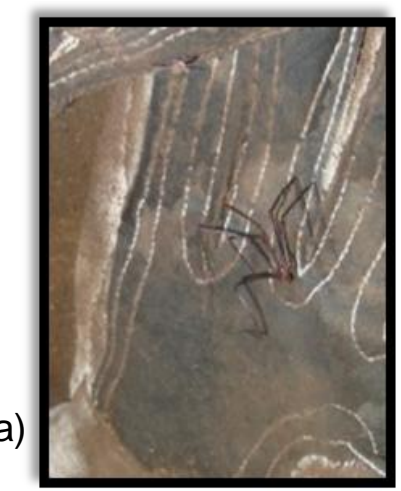

(3a)

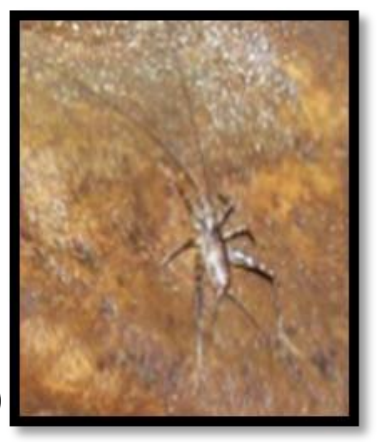

(3b)

Imagen2a: "Cueva del Lago". Esta cueva presenta un gran número de pasajes o túneles y su centro un hermoso lago que es alimentado por distintas quebradas proveniente de los pasajes de la misma.

Imagen 3a: Un Arácnido del Orden Opiliones presentaba ocelos reducidos y vellosidades pronunciadas en su céfalotórax.

Imagen 3b: Un insecto sin ojos, pero con antenas Filiformes de gran longitud.

Continuando con lo anterior el grupo investigador decidió adentrarse aún más a la cueva y fue gracias a ello que el momento esperado por todos tuvo lugar, cuando desde los distintos puntos cardinales se escucharon pequeños y constantes ruidos semejantes a chillidos, más el aleteo vertiginoso que pronosticaba el hallazgo de murciélagos al pasar justo por los rostros y cuerpos, sin poderlos apreciar pues era necesario apagar las linternas y evitar el ruido para poderlos capturar.

Es pertinente decir que las herramientas empleadas en esta práctica espeleológica fueron parte de la dotación, linternas de cabeza, con luz blanca y Jamas de gran tamaño elaboradas de manera artesanal por INDUCIENCIAS para poder facilitar la captura y estudio de los murciélagos, además del equipo de seguridad: guantes, gafas y sogas de protección. La acción de las capturas se tornó difícil por el espacio en puntos de la cueva, muchas veces reducido y lleno de rocas de gran tamaño pero con formación caliza lo que impedía posarse sobre ellas por lo cual no podían considerarse firmes ni sitios seguros.

Ya con los equipos y la información adecuada para la captura de los murciélagos dentro de las cueva solo faltaba tener un ejemplar que diera pistas de especímenes de murciélagos encontrados allí, y evidenciar posiblemente hábitos alimenticios y comportamentales. El primer murciélago colectado por el grupo investigador fue un macho caracterizado por tener un color negro, una longitud en el ala de $35 \mathrm{~cm}$, y una nariz en forma alargada y con bordes semejantes a los de una pluma, y esto último fue fundamental para definir los caracteres taxonómicos propios de la especie Anoura geoffroyi, (Gray, 1838) de la cual es representante (observar imagen 4a y4b), también se evidenció éste como un organismo Nectarívoro debido a su larga lengua que podía 
tomar forma curvada para la succión del néctar. Por otra parte, al ser un organismo que habita en la oscuridad, sus oídos son receptores especializados en ciertos ruidos, ya que la comunicación de los quirópteros se hace por medio de un sonar adecuado, o ecolocación a sus chillidos característicos y es por esto que las voces del grupo investigador aparentemente parecían aturdirlo y desorientarlo.

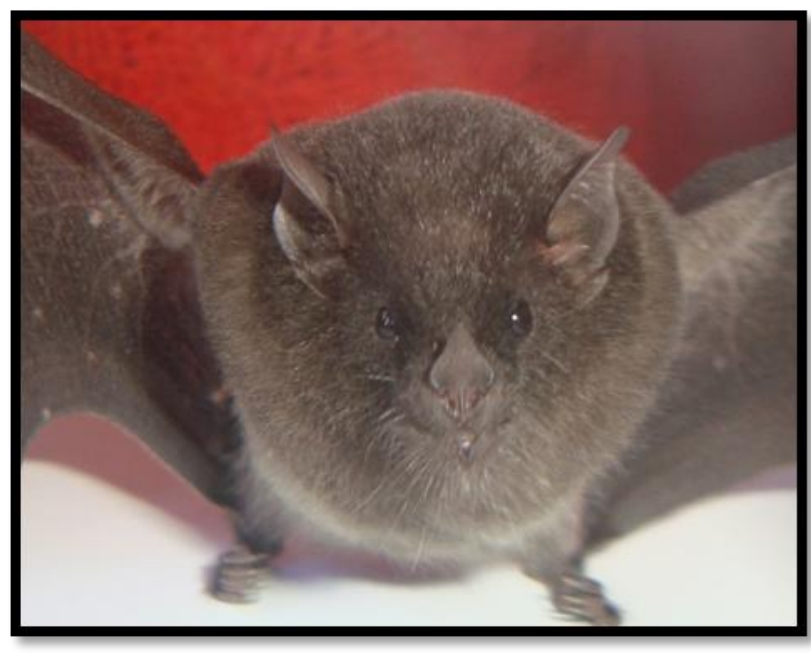

(4b)
Reino: Animalia

Phylum: Chordata

Clase: Mammalia

Orden: Chiroptera

Familia: Phyllostomidae

Subfamilia: Glossophaginae

Género: Anoura

Especie: $A$. geoffroyi (Gary, 1838)

(4a)

Imagen 4a: Anoura geoffroyi. Primer individuo colectado en la "Cueva del lago", fue un macho caracterizado por tener un color negro, una longitud en el ala de $35 \mathrm{~cm}$, y una nariz en forma alargada y con bordes semejantes a los de una pluma.

Imagen 4b: ficha taxonómica de Anoura geoffroyi. Determinado por García Mario, miembro de la empresa Bioimpacto S.A.S.

De igual manera, mientras se realizaba el análisis de las estructuras morfológicas que conformaban las alas se encontraron garrapatas (Orden: Acari) adheridas a las mismas y debido a esto, la inferencia a la cual se llegó en el grupo es que eran ectoparásitos que se alimentaban de la sangre que circulaba por las venas del murciélago. Después de hacer la descripción y tomar las respectivas fotos del organismo se decidió dejarlo en libertad para así continuar el recorrido en la segunda cueva. Este tipo de procedimiento fue realizado en cada cueva visitada y en lo posible las capturas hechas solo se hacían para la toma de datos y nunca para sacrificar los especímenes.

La segunda cueva visitada en la Vereda Canavita fue la "Jama-Cueva" denominada así por la fascinación que causaron las Jamas entre el grupo INDUCIENCIAS y por la utilidad que éstas tenían en la colecta de murciélagos (observar imagen 5a y 5b). Esta cueva al Igual que la anterior presentaba unas características propias que permitían la permanecía de murciélagos tales como la humedad relativa del $100 \%$, la escases de luz y la presencia del hongo Histoplasma capsulatum, que como se mencionó anteriormente está muy ligado al hábitat de los mismos. 


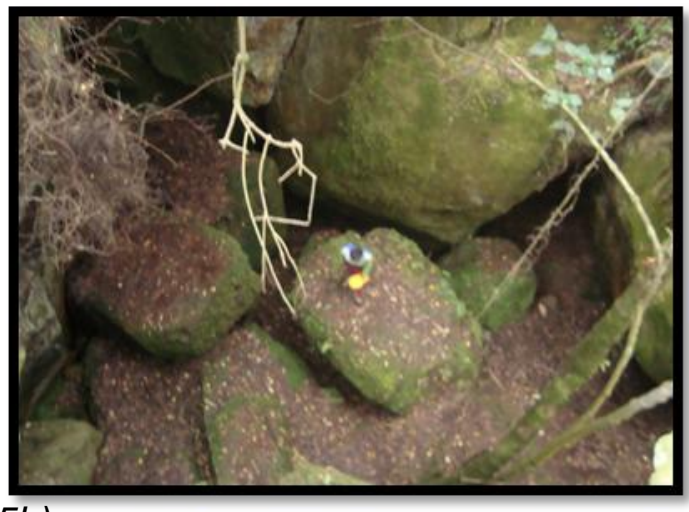

(5b)

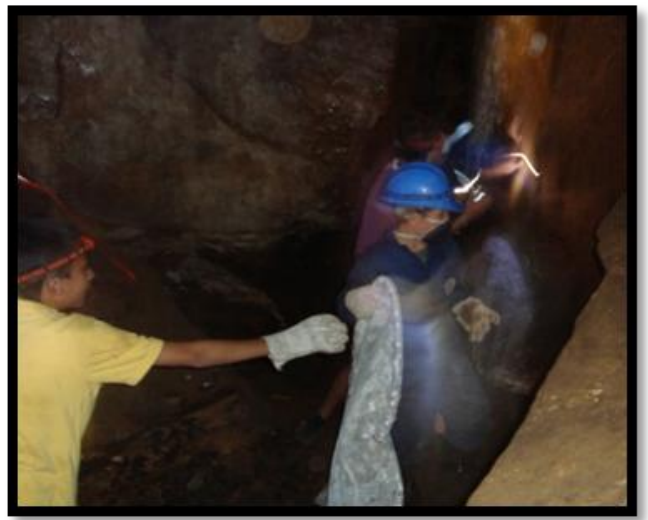

Imagen 5a: vista superior de la "Jama cueva”

Imagen 5b: vista interna de la "Jama Cueva" en esta fotografía es posible observar a los miembros de INDUCIENCIAS con Jamas para la captura de murciélagos

Dentro de la cueva el grupo tuvo la oportunidad de observar a un gran número de murciélagos, ya que estos se encontraban reunidos en una de las cámaras de la cueva y a su vez lograron identificar que estaba en época de apareamiento (mes de abril) debido a que habían algunos de ellos copulando, además de observar algunos ritos propios de este acto (observar imagen $6^{a}$ ), de otra manera los quirópteros encontrados allí pertenecían a una especie diferente (aun sin determinar), pero que son representantes de la Familia Phyllostomidae y este en especial se caracterizó por la presencia de cola en el patagio, quijada prominente a manera de lobo y bastante pelo (observar imagen 6b).

(6a)

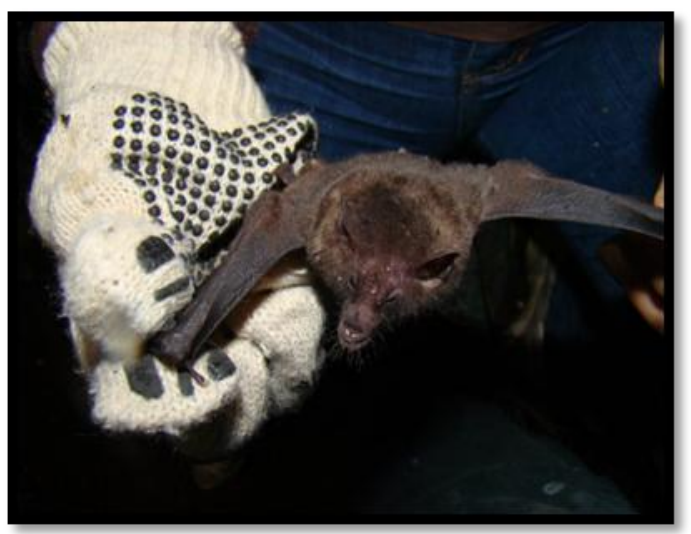

(6b)

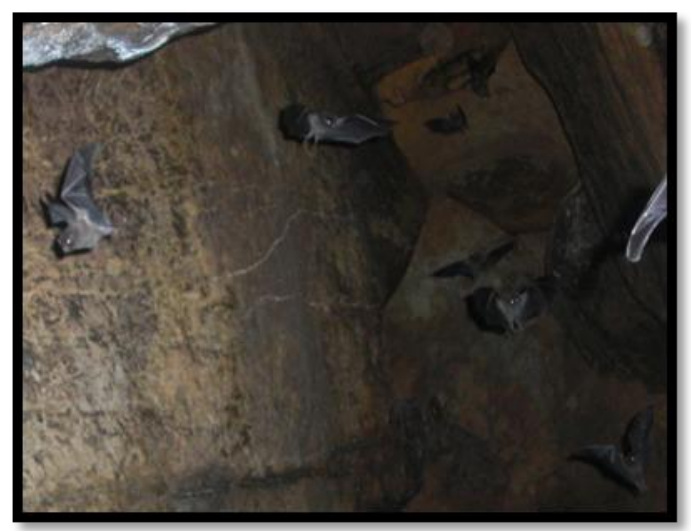

Imagen 6a: visita a la "Jama cueva", donde fue posible evidenciar los ritos de apreamiento de los murcielagos

Imagen 6b: segunda captura de un murciélago de la familia Phyllostomidae, aunque la asesoría de García Mario en mastozoología demoro que este individuo pertenece a otro género y especie diferente a Anoura geoffroyi

Ya por último la tercera cueva visitada tenía el nombre de La cueva del Colorado y se encuentra ubicada en la vereda La Fuente y es conocida por sus habitantes debido a una leyenda propia de la región (observar imagen $7 a$ y $7 b$ ), pero en este sitio no se encontró ningún murciélago por el contrario se observó cómo la prácticas humanas deterioran el 
hábitat de estos organismos, ya que las condiciones necesarias para su existencia se encontraban totalmente modificadas debido al constate depósito de basuras y de la contaminación de la fuente hídrica que por allí fluye, eso sin contar la destrucción del busque nativo para la introducción de animales de pastoreo.

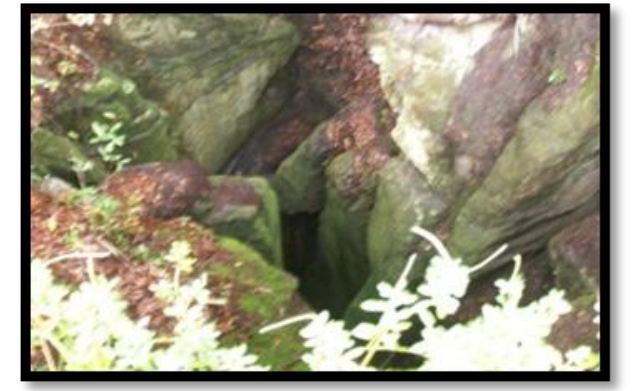

(7a)

Imagen 7a: vista superior de la cueva del colorado

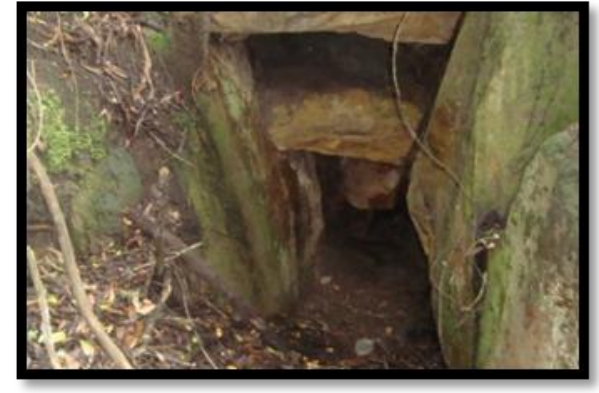

(7b)

Imagen 7b: pasaje más asequible de la cueva del colorado para el grupo investigador INDUCIENCIAS

\section{LOS ESTUDIANTES Y LA LABOR PEDAGOGICA.}

Después de las salidas de campo solo quedaba hacer un repaso de lo que fue la labor de investigación de INDUCIENCIAS y de cómo su trabajo se caracterizó por entusiasmo y buena disposición para cada actividad planteada, participaron 14 estudiantes que pertenecían a diferentes grados de educación básica secundaria y educación media, se vincularon al proyecto libremente; cinco de ellos ya pertenecían al grupo ecológico Institucional, Laura Daniela Bojacá de grado séptimo, Jessicca Andrea Renteria, Freddy Sanchez, Oscar Puentes, y Carolina Bojacá de grado décimo, con ellos surgió la idea de estudiar la "biodiversidad" del municipio de Tocancipá, 8 más se integraron en el grupo por petición de ellos mismos durante las clases de biología o porque tuvieron conocimiento de lo que se hacía al interior del grupo, así :Lady Cuervo, Miguel Garzón (promotor de la pregunta ¿hay murciélagos en Tocancipá?) Ximena Duran, Asdrubal Díaz, y Jonathan Primiciero de décimo y octavo grados se convirtieron rápidamente en los lideres investigadores y participantes activos del proyecto los cuatro restantes se retiraron por cambio de domicilio y problemas de salud.

Lo que más les llamó la atención fue el hecho de realizar cosas diferentes a lo tradicional en el aula de clases, como realizar las salidas de campo con índole investigativo, representar el colegio dando a conocer el producto de su trabajo, y aprender en otro contexto diferente al salón de clases, sin obtener una calificación por ello, esto pudo crear desmotivación y desconcierto en algunos momentos ya que los alumnos están condicionados a recibir buenas calificaciones a través de evaluaciones escritas, tareas y cuestionarios escritos, es por esta razón que realizar actividades diferentes fuera del aula que no implican actos como copiar de un texto, resolver talleres, o estudiar para una previa, resulta ser algo inquietante que pone al estudiante en un ambiente de expectativa y crea espacios para demostrar conocimientos de una forma menos tradicional. Pese a que este trabajo se desarrolló en tiempos extra clase, en horas y días diferentes a los de su horario habitual, con una dinámica diferente por su misma naturaleza, los estudiantes participantes realizaron las actividades con agrado, gusto y mucho entusiasmo al saber que estaban haciendo algo sin precedentes en la localidad donde habitan y al final del proceso recibieron todo el reconocimiento y mérito producto de su esfuerzo. 
El estudiante a través de este trabajo logró estructurar un poco más su pensamiento productivo, al establecer relaciones entre los resultados obtenidos y los problemas planteados en las preguntas, formulando nuevos cuestionamientos de investigación con un grado de profundidad mayor, lo que demuestra un progreso en el razonamiento para producir ideas más claras que conllevan a la realización de nuevas propuestas investigativas en donde los protagonistas son ellos mismos con un alto sentido de responsabilidad en la conservación de los recursos de su entorno.

La transformación del pensamiento en la actividad del aula se ha visto marcada en los estudiantes que hicieron parte de este proyecto en comparación con los que solo son observadores del proceso, se muestran más analíticos, consultan y cuestionan la ciencia, proponen alternativas y sus razonamientos concretos ponen en evidencia el efecto que tiene acercarse al quehacer científico a través de esta propuesta metodológica para abarcar una investigación sacada del aula, puesta en práctica fuera de ésta y de regreso a ella para demostrar que las estructuras cognitivas pueden ser ampliadas, modificadas y mejoradas notablemente.

\section{EL APOYO.}

Fue así como los orientadores de la investigación: su profesora de clase de biología y su amigo estudiante de licenciatura en Biología, emprenden con el grupo un camino nada fácil en la consecución de su objetivo realizar un estudio para describir inicialmente la fauna y flora del municipio de Tocancipá, con escasos recursos y algunos pequeños aportes que obtuvo el proyecto por parte del programa ONDAS de COLCIENCIAS (observar imagen 8a), la Gobernación de Cundinamarca y la UNIVERSIDAD PEDAGOGICA NACIONAL, en virtud a esto se lograron resultados en la determinación algunas de las especies de los quirópteros encontrados, de la mano de Mario A. García Mora, egresado de la Universidad Nacional de Colombia y actual miembro de la empresa BIOIMPACTO, quién estableció las pautas para la captura de murciélagos, la determinación de las especies, el desplazamiento en las zonas de estudio y los cuidados requeridos al interior de la cuevas (observar imagen 8b), el resultado fue obtener un gran avance en el proceso del proyecto, recibir de manera verbal la categoría de Pioneros en el departamento de Cundinamarca, por parte de los directores del PROGRAMA ONDAS.

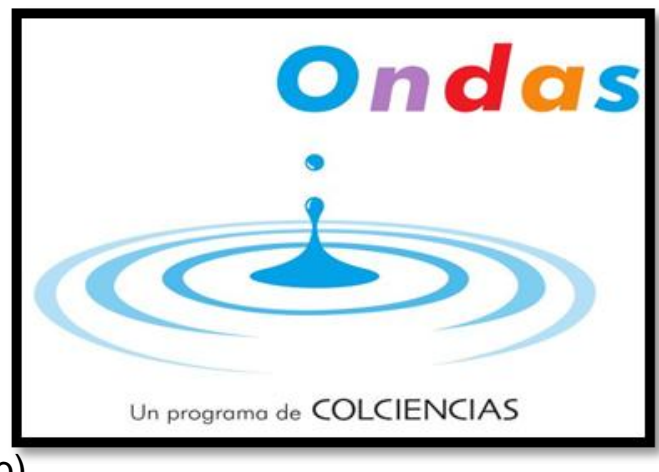

(8a)

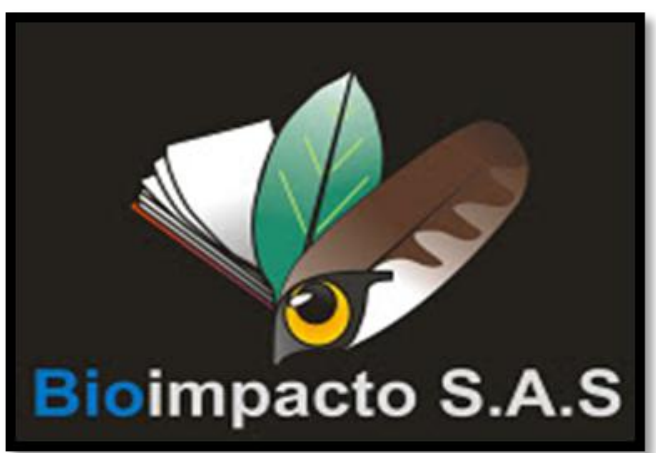

$(8 b)$

Imagen 9a: Imagen de Ondas- de Colciencias, quién aporto en gran parte el presupuesto empleado en la investigación.

Imagen 9b: Imagen de la empresa Bioimpacto, a la cual pertenece Mario A. García quién asesoro la colecta y determinación de los murciélagos. 
Los conocimientos adquiridos se divulgaron en televisión local y sin lugar a dudas la publicación del RAE del proyecto en la revista: Proyectos de Investigación Ondas Cundinamarca, 2009-2010 de Colciencias, Universidad Pedagógica Nacional, y CIUP. La labor en la búsqueda del conocimiento, la admiración en las personas que escuchan hablar a los niños y adolescentes de la presente investigación, la labor de sus docentes, en la disciplina, constancia y esfuerzos propios para lograr cierto grado de rigurosidad en los conocimientos adquiridos, los hallazgos encontrados, la toma de datos, el cuidado de las especies, la protección de las mismas, la descripción de las zonas de estudio, las condiciones climáticas, el análisis de resultados y la solución a las principales preguntas planteadas por los niños en las clases de biología objeto de estudio del presente proyecto y las preguntas que surgieron en el trascurso de la investigación, hicieron que el naciente grupo denominado INDUCIENCIAS adquiriera el valor que hoy tiene no solo como su consolidación, sino como un hecho a través del cual se dio cabida a una experiencia significativa, no solo para satisfacer las expectativas de sus docentes y colaboradores sino de toda la comunidad aledaña y la apertura a formar un grupo, quizás aún mayor de investigación en Biología de la Conservación a nivel estudiantil para las presentes y futuras generaciones en el Departamento de Cundinamarca y de Colombia.

\section{LAS PROYECCIONES}

A lo largo de la investigación, se logró establecer que las temáticas que fundamentaban el trabajo con los estudiantes, también eran parte de la construcción de un conocimiento significativo, debido a que el interés por conocer y describir el entorno que les rodea es la base la interiorización del conocimiento a nivel metacognitivo, además, este tipo de ejercicios como lo son, la formulación de preguntas y prácticas en campo, aportan el fortalecimiento de las temáticas que no pueden ser desarrolladas en el aula clase.

Por otra parte con este estudio se logró un acercamiento al concepto de biodiversidad en dos aspectos fundamentales; primero, el trabajo ejecutado permitió construir un conocimiento más elaborado sobre la importancia del estudio de especies que no son reconocidas socialmente, puesto que sus relaciones con el medio son concebidas como algo ajeno y sin relevancia en la cotidianidad de la personas que conforman la comunidad. Segundo, es un acercamiento al concepto de biodiversidad porque la investigación sigue en curso, para abarcar en el año 2011 nuevas preguntas de investigación relacionadas con la descripción del paisaje, elaboración de mapas, estudio de la variabilidad genética en la población de la familia Phyllostomidae, determinación tanto de especies vegetales como animales asociadas a esta familia, además de la divulgación de la importancia de conservar diferentes especies de murciélagos, ya que desde el estudio de la conservación, estos son considerados especies clave en el mantenimiento de los ecosistemas a los que pertenecen. 
Bio-grafia: Escritos sobre la Biologia y su Enseñanza Vol. 4 No7. ISSN 2027-1034.

Segundo Semestre de 2011. P. p.121-131

De esta manera solo podemos decir que el interés de los docentes y la participación de los estudiantes son la base de la construcción del saber Biológico puesto que este proyecto logra demostrar que el trabajo en equipo puede hacer la diferencia en la enseñanza de la Biología en las instituciones Educativas del país.

"No podremos cambiar el mundo, pero hemos logrado transformar nuestras vivencias"

C, Possos; C, Martín (2011) 